\title{
Assessment of domestic violence against elderly people in Mansoura city .
}

\author{
Nora El-sayed Mahmoud ${ }^{1}$, Prof. Nagat Said Habib ${ }^{2}$, Assist.Prof. Nagla Ibrahim \\ Mohamed Gide ${ }^{3}$, Assist. Prof. Maha Moussa Mohamed Moussa ${ }^{4}$
}

B.Sc, Mansoura University ${ }^{1}$, Professor of Community Health Nursing, Faculty of Nursing-Cairo University ${ }^{2}$, Assistant Professor of Family and Community Health Nursing, Faculty of Nursing - Port Said University ${ }^{3}$, Assistant Professor of Family and Community Health Nursing, Faculty of Nursing - Port Said University ${ }^{4}$

\begin{abstract}
Background: Domestic violence occurs when one person in an intimate relationship behaves in a way that causes fear or harm to another person. Domestic violence includes emotional, physical, sexual, neglect and financial exploitation.. The aim: To assess domestic violence against elderly people in Mansoura city. Research design: A descriptive cross-section research design was utilized. Settings: The study conducted in rural area, at outpatient clinics that located in a central hospitals of three districts: Menait ElNasr, Sherbien, and Aga; these districts were randomly selected from fifteen districts in Mansura city. Sample type: A covenant sample was used. Sample size: The sample consisted of 394 elderly. Tools: Interviewing questionnaire sheet and elderly abuse assessment instrument were used to collect the study subjects' data. Results: The results revealed that the elderly people were exposed to physical violence (13.7\%), emotional violence $(45.9 \%)$, neglect (13.7\%), financial exploitation $(10.2 \%)$, and sexual violence (2.8\%). Conclusion: the elderly people have lack of knowledge about domestic violence and the most common type of elderly domestic violence was emotional. Recommendation: the study recommended to increase the knowledge of the community about elderly domestic violence through mass media.
\end{abstract}

Key word: financial exploitation, physical violence, psychological violence 1, selfneglect , 


\section{INTRODUCTION}

Domestic Violence is a growing worldwide problem within households and in public spheres. Violence has an adverse impact on society. It is estimated to cost billions of dollars per year these costs include direct medical care and rehabilitation, thus they have a notable impact on family and the larger community. Domestic violence is an epidemic in societies with drastic and negative effects on individuals, families, and communities (Puchala, et al. ,2010).

There are three basic categories of violence ; domestics, institutional and selfneglect .Domestic elderly violence refers to the maltreatment of an older person residing in his or her own home or the home of a caregiver. Institutional violence occurs in residential facilities while self-neglect refers to neglect inflicted when the safety or health of elderly people is threatened by their living alone( Administration on Aging (AOA),2012) .

Domestic violence is a pattern of behavior which involves violence by one person against another in a domestic setting (Natalie, 2013). Elderly domestic violence is a single, repeated act and lack of appropriate action occurring within any relationship where there is an expectation of trust which causes harm and distress to an older person (Killick, et al., 2015) . Domestic violence can have various forms such as physical, emotional, sexual, neglect and financial violence (World of Health Organization (WHO),2011).

\section{Significance of the study:}

The present study was assessed the domestic violence against elderly people in Mansoura city. The most common type of domestic violence against elderly people in rural areas was neglect (42.4\%) followed by physical abuse (5.7\%), psychological abuse (5.1\%) and financial abuse (3.8\%) (Abdel Rahman, \& El-Gaafary, 2012). Domestic violence against elderly people is an important cause of morbidity and mortality. Community health nurses as well as family physicians are well placed to identify mistreatment of seniors their actual rates of reporting violence are lower than those in other professions. This might be improved by an understanding of the range of acts that constitute elder violence and what signs and symptoms seen in the health care outpatient clinics might suggest domestic 
violence against elderly people (Robinson, et al., 2014). So that to shed the light on such problem against the Egyptian rural elderly community.

\section{AIM OF STUDY:}

The aim of the present study was to assess domestic violence against elderly people in Mansoura city.

\section{Research questions:}

1. What are the forms of domestic violence against elderly people ?

2. What are the factors related to domestic violence ?

\section{SUBJECTS AND METHODS:}

\section{Technical Design:}

-Research design: A descriptive cross-section research design was utilized in this study to achieve the study aim.

- Setting: The study conducted in rural area, at outpatient clinics that present in a central hospital of three district : Menait El-Nasr, Sherbien ,Aga district which selected randomly from fifteen district in Mansura city .

\section{Sampling design:}

Target Sample: The study sample included elderly people.

Sampling technique: A covenant sample was used to select the required sample.

\section{Tools of data collection:-}

Two different tools were used in the study, and collected by the researcher .

Tool (1): Interviewing Questionnaire sheet (Appendix I): It was developed by the researcher based on reviewing related literatures, it includes three parts:

Part I: which include two sections :-

1- socio-demographic characteristics, it is contain 14 item such as (age, gender, level of education, marital status ,family member living with him / her and sources of income......ect ) . 
2- social activities :- it include 3 questions about social activities such as ( visiting relatives, attending social occasions and entertainment $\mathrm{TV} /$ radio ).

Part II: which include medical history, it contain 9 questions about presence of medical problems such as (diabetes, hypertension, heart disease), surgical operation and medication availability.

Part III: assessment of elderly violence condition, it included questions 1-3 about knowledge of the elderly about violence, questions 4-39 about elderly exposing to violence ( physical abuse questions 4-10, emotional abuse questions 11-17, neglect questions $18-28$, financial abuse from 29 -34 and sexual abuse questions $35-39$ ).

\section{Tool (2): The Elder Abuse Assessment Instrument (Appendixes II ) :}

The Elder Abuse Assessment Instrument (EAI) was composed and validated by Fulmer (2003)and adapted by the researcher. It is a 41 items screening tool comprising 5 subscales for identifying general assessment, signs of abuse ,neglect, exploitation and abandonment.

\section{Scoring system:-}

The scale was completed the items on a 3 point Likert scale what is the answers of the patients. The first part was categorized as general assessment, scored as very good (+60 $\%)$, good $(50-<60 \%)$, and poor $(<50 \%)$. The other subscale of the tool was scored as definite evidence (_> $50 \%$ ), no evidence (zero), and possible evidence $(<50 \%)$.

\section{II- Administrative Design and Ethical Considerations:}

An official letter addressed from the dean of the Port Said Faculty of Nursing to the study setting medical and nursing directors to obtain their permission for conducting the study. It explained the study objective and its procedures. Moreover, An official letter from the Faculty of Nursing present with the researcher to obtain permission of present research from the manger of each hospital .

\section{III-Operational design:}

Preparatory phase: During this phase, the researcher reviewed the literature related to the study subject using paper and electronic sources both locally and internationally. This helped in the selection and preparation of the data collection tools. 
Content validity: Upon preparation of the preliminary forms of the tools, they were presented to a panel of 5 experts for face and content validation. They were from Nursing community department from Port Said Faculty of Nursing. They assessed the tool for clarity, relevance, comprehensiveness, understanding and ease for implementation. According to their opinions, minor modifications were applied. The tools were modified according to their recommendations and suggestions.

Testing reliability: Cronbach \& coefficient was calculated to assess the reliability of the tool through its internal consistency Cronbach \& value was $91 \%$.

Reliability of Elder assessment instrument for exposure to abuse among the studied elders:-

\begin{tabular}{|c|c|}
\hline Elder assessment instrument & Cronbach \& value \\
\hline General assessment score & 0.973 \\
\hline Possible abuse score & 0.819 \\
\hline Possible neglect score & 0.905 \\
\hline Possible exploitation score & $\mathbf{0 . 8 7 3}$ \\
\hline Possible abandonment score & $\mathbf{0 . 9 4 2}$ \\
\hline
\end{tabular}

Pilot study: A pilot study was carried out 40 elderly from the study setting, representing $10 \%$ of the sample size. The purposes of the pilot study were to ascertain the clarity and feasibility of the tools, and to detect any possible problems concerning data collection tools that might face the researcher and interfere with data collection. It also helped to identify the suitable time and place for data collection, and to estimate the exact time needed for data collection.

The field work: Data was collected from the selecting settings by the researcher using the pre constructed tool. The questionnaire sheet was filled in by the researcher while asking the elderly people during their attending in the hospital, purpose of the study was explained prior to get the questionnaire sheet. Data collection was implemented through three months from the first of March to the end of May 2015, two day for every hospital per week. The time for structured interview sheet varied between $20-30$ minutes. The researcher make from five to six interviews every day. 
VI-Statistical Analysis: The raw data were coded and entered into SPSS system files (SPSS package version 18). Analysis and interpretation of data were conducted.

The following statistical measures were used:

Descriptive statistics including frequency, distribution, mean, median, standard deviation and inter-quartile range were used to describe different characteristics. Kolmogorov Smirnov test was used to examine the normality of data distribution. Univariate analyses including: Chi-Square test, Monte Carlo test and Fisher's Exact test were used to test the significance of results of qualitative variables. The significance of the results was at the $5.0 \%$ level of significance.

\section{RESULTS:}

Table (1): shows that about one-third $(34.5 \%)$ of the studied sample being in the age group of (65 to $<70$ years), while those aging 80 or more constituted $6.6 \%$ with mean age (68.8 \pm 5.8$)$. More than half of them $(53.0 \%)$ were males. Regarding the level of education, about three-fifth (58.6\%) of the sample were illiterate, and who can read and write represented about one-fourth $(25.6 \%)$, while only $(2.4 \%)$ of the total sample having University education. As for marital status, married people constituted about three-fifth (58.6\%), divorced constituted (1.5\%) of the sample. Regarding the presence of offspring, the majority (92.6\%) of the sample had offspring and about three-fifth (60\%) of those offspring had basic education or less. Regarding to house hold member, more than half (55.6\%) of the sample were live with their families, while the lowest percentages of the sample $(0.3 \%)$ were living in elders-care home. As for, having monthly fixed salary, about four-fifth $(81.7 \%)$ of total sample having fixed salary and about two-fifth $(41.9 \%)$ having insufficient monthly salary.

Table (2): shows that about two-fifth of the study sample (42.1\%) had knowledge that domestic violence was "hitting" while the least (3.3\%) of the study sample considered that violence was "sexual contact". Considering the type of domestic violence, this table shows that physical, emotional, and neglect $(42.4 \%, 35.8 \% \& 33.2 \%)$ respectively were known among elderly, while about one-fourth $(24.9 \%)$ of them didn't know. 
Table (3): shows the relation between studied elderly reported exposing to physical domestic violence and their socio-demographic characteristics. The table reveals that there were statistical significant associations were detected between exposure to physical violence and number of children and having chronic diseases ( $\mathrm{p}$-value $=0.004$ and 0.016 respectively).

Table (4): represents the relation between the studied elderly reported exposing to emotional domestic violence and their socio-demographic characteristics. This table shows that there were statistical significant associations detected between elderly who were exposed to emotional violence and all items of their socio-demographic characteristic except the item of "have enough salary" it didn't show statistically significant association ( $\mathrm{p}$-value=0.339).

Table (5): illustrates the relation between the studied elderly reported exposing to neglect and their socio-demographic characteristics. This table shows there were statistical significant associations detected between marital status, number of children, accompanying household, have enough salary and chronic diseases ( $\mathrm{p}$-value $=0.002$, $0.011,0.0001,0.021$ and 0.043 respectively).

Table (6): discloses the relation between the studied elderly reported exposing to financial domestic exploit and their socio-demographic characteristics. This table shows that there were a statistical significant relations detected between exposure to financial exploit and marital status, and accompanying house hold (p-value $=0.041$ and 0.038 respectively).

Table (7): demonstrates the relation between the studied elderly reported exposing to sexual domestic violence and their socio-demographic characteristics. This table shows there were a statistical significant relations detected between elderly exposed to sexual abuse and gender and marital status ( $\mathrm{p}$-value $=0.019$ and 0.0001 respectively). 
Table (1): Percentage distribution of studied elderly according to their sociodemographic characteristics at outpatient clinic $(n=394)$

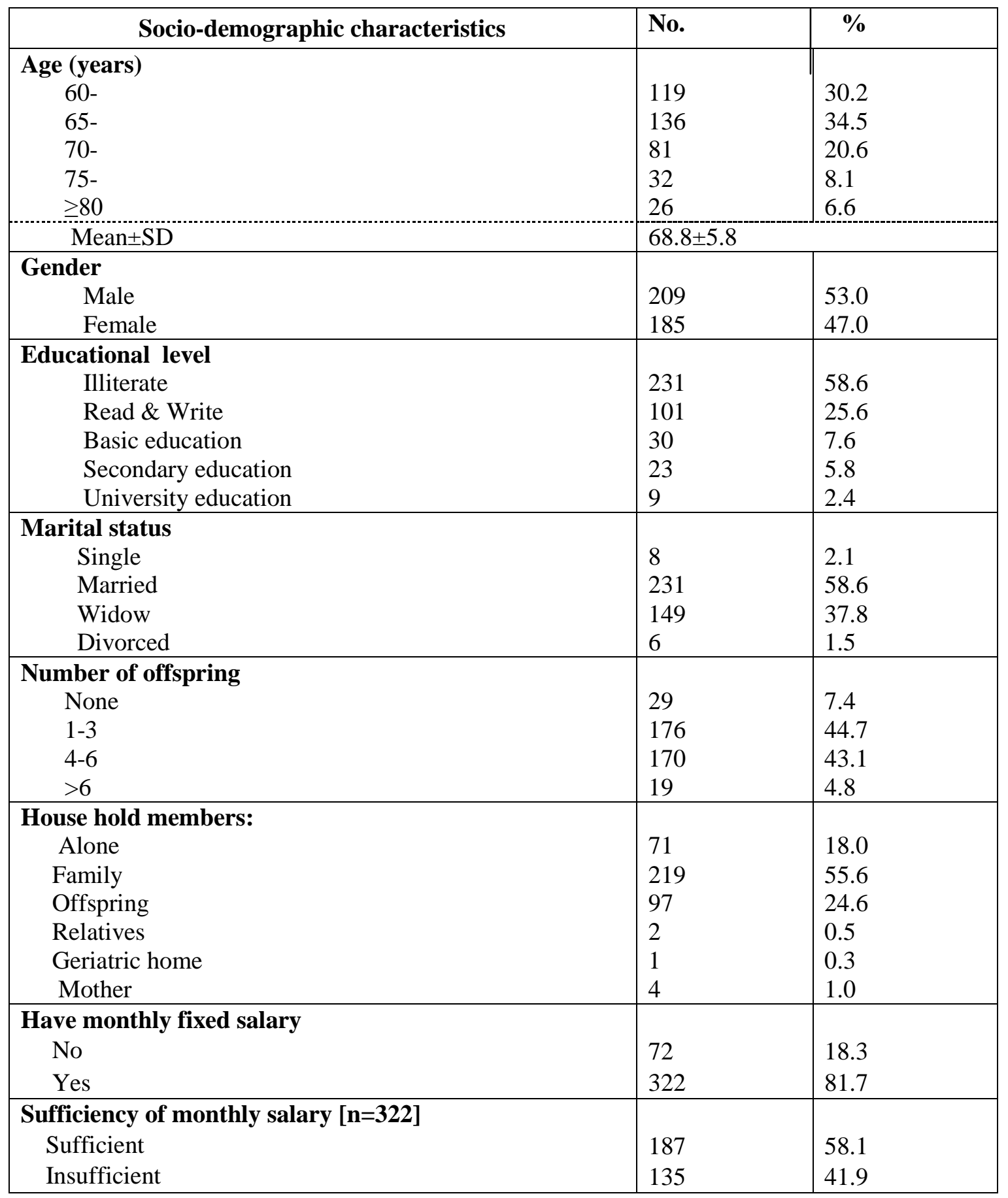

* Numbers are not mutually exclusive 
Table (2): Percentage distribution of the studied elderly's knowledge about domestic violence.

\begin{tabular}{|c|c|c|}
\hline Elderly's knowledge & No. & $\%$ \\
\hline \multicolumn{3}{|l|}{ * Definition of domestic violence } \\
\hline Hitting & 166 & 42.1 \\
\hline Bad words & 144 & 36.5 \\
\hline Sexual contact & 13 & 3.3 \\
\hline Don't care & 128 & 32.5 \\
\hline Taking money & 42 & 10.7 \\
\hline Don't know & 98 & 24.9 \\
\hline \multicolumn{3}{|l|}{ *Types of domestic violence } \\
\hline Physical & 167 & 42.4 \\
\hline Emotional & 141 & 35.8 \\
\hline Neglect & 131 & 33.2 \\
\hline Financial & 44 & 11.2 \\
\hline Sexual & 14 & 3.6 \\
\hline Don't know & 98 & 24.9 \\
\hline \multicolumn{3}{|l|}{ *Reasons for domestic violence } \\
\hline Offspring virgules & 154 & 39.1 \\
\hline Offspring maltreatment & 160 & 40.6 \\
\hline Poor economic status & 117 & 29.7 \\
\hline Poor religious & 95 & 24.1 \\
\hline Speed pattern of life & 139 & 35.3 \\
\hline Offspring disobedience & 29 & 7.4 \\
\hline
\end{tabular}


Table (3): Relation between the studied elderly reported exposing to physical domestic violence and their socio-demographic characteristics $(n=394)$

\begin{tabular}{|c|c|c|c|c|c|}
\hline \multirow{3}{*}{ Socio-demographic characteristics } & \multicolumn{4}{|c|}{ Exposure to physical domestic violence } & \multirow{3}{*}{$\begin{array}{l}\text { Significance } \\
\text { tests }\end{array}$} \\
\hline & \multicolumn{2}{|c|}{ No $(n=340)$} & \multicolumn{2}{|c|}{ Yes $(n=54)$} & \\
\hline & No. & $\%$ & No. & $\%$ & \\
\hline \multicolumn{6}{|l|}{ Age (years) } \\
\hline $60-$ & 102 & 30.0 & 17 & 31.5 & \\
\hline $65-$ & 119 & 35.0 & 17 & 31.5 & $X^{2}=4.878$ \\
\hline 70- & 66 & 19.4 & 15 & 27.7 & ${ }^{{ }^{M} C_{p}=0.302}$ \\
\hline 75- & 31 & 9.1 & 1 & 1.9 & \\
\hline$\geq 80$ & 22 & 6.5 & 4 & 7.4 & \\
\hline \multicolumn{6}{|l|}{ Gender } \\
\hline Male & 179 & 52.6 & 30 & 55.6 & $X=0.158$ \\
\hline Female & 161 & 47.4 & 24 & 44.4 & \\
\hline \multicolumn{6}{|l|}{ Educational level } \\
\hline Illiterate & 201 & 59.1 & 30 & 55.6 & \\
\hline Read \& Write & 86 & 25.3 & 15 & 27.7 & $X^{2}=1.377$ \\
\hline Primary education & 27 & 7.9 & 3 & 5.6 & ${ }^{\mathrm{MC}} \mathrm{p}=0.867$ \\
\hline Secondary education & 19 & 5.6 & 4 & 7.4 & \\
\hline University education & 7 & 2.1 & 2 & 3.7 & \\
\hline \multicolumn{6}{|l|}{ Marital status } \\
\hline Married & 198 & 58.3 & 33 & 61.1 & \\
\hline Divorced/single & 13 & 3.8 & 1 & 1.9 & $\begin{array}{l}\mathrm{A}-0.070 \\
\mathrm{MC}_{\mathrm{n}}-0.746\end{array}$ \\
\hline Widow & 129 & 379 & 20 & 370 & \\
\hline \multicolumn{6}{|l|}{ Number of children } \\
\hline No children & 29 & 8.5 & 0 & 0.0 & \\
\hline From 1-3 & 155 & 45.6 & 21 & 38.9 & $\mathrm{MC}_{\mathrm{n}-0.004 *}$ \\
\hline From 4-6 & 144 & 42.4 & 26 & 48.1 & \\
\hline More than 6 & 12 & 3.5 & 7 & 13.0 & \\
\hline Accompanying house hold & & & & & \\
\hline Alone & 60 & 17.6 & 11 & 20.4 & $\mathrm{D}-0.620$ \\
\hline Not alone & 280 & 82.4 & 43 & 79.6 & \\
\hline Have enough salary & {$[\mathrm{n}=280]$} & & {$[\mathrm{n}=42]$} & & $x^{2}-3269$ \\
\hline Enough & 168 & 60.0 & 19 & 45.2 & $n-0.071$ \\
\hline Not enough & 112 & 40.0 & 23 & 54.8 & \\
\hline chronic diseases & & & & & $\mathrm{X}^{2}=5.812$ \\
\hline No & 64 & 18.8 & 3 & 5.6 & $\mathrm{p}=0.016^{*}$ \\
\hline Yes & 276 & 81.2 & 51 & 94.4 & \\
\hline
\end{tabular}


Table (4): Relation between the studied elderly reported exposing to emotional domestic violence and their socio-demographic characteristics $(n=394)$

\begin{tabular}{|c|c|c|c|c|c|}
\hline \multirow{3}{*}{$\begin{array}{l}\text { Socio-demographic } \\
\text { characteristics }\end{array}$} & \multicolumn{4}{|c|}{ Dealing in an inappropriate way } & \multirow{3}{*}{$\begin{array}{l}\text { Significance } \\
\text { tests }\end{array}$} \\
\hline & \multicolumn{2}{|c|}{ No $(n=213)$} & \multicolumn{2}{|c|}{ Yes $(n=181)$} & \\
\hline & No. & $\%$ & No. & $\%$ & \\
\hline $\begin{array}{l}\text { Age (years) } \\
60- \\
65- \\
70- \\
75- \\
\geq 80 \\
\end{array}$ & $\begin{array}{l}71 \\
83 \\
37 \\
14 \\
8\end{array}$ & $\begin{array}{l}33.3 \\
39.0 \\
17.4 \\
6.5 \\
3.8\end{array}$ & $\begin{array}{l}48 \\
53 \\
44 \\
18 \\
18 \\
\end{array}$ & $\begin{array}{l}26.5 \\
29.4 \\
24.3 \\
9.9 \\
9.9 \\
\end{array}$ & $\begin{array}{l}X^{2}=13.504 \\
p=0.009 *\end{array}$ \\
\hline $\begin{array}{l}\text { Gender } \\
\text { Male } \\
\text { Female } \\
\end{array}$ & $\begin{array}{l}142 \\
71\end{array}$ & $\begin{array}{l}66.7 \\
33.3 \\
\end{array}$ & $\begin{array}{l}67 \\
114 \\
\end{array}$ & $\begin{array}{l}37.0 \\
63.0 \\
\end{array}$ & $\begin{array}{l}X^{2}=34.537 \\
p<0.0001^{*}\end{array}$ \\
\hline $\begin{array}{l}\text { Educational level } \\
\text { Illiterate } \\
\text { Read \& Write } \\
\text { Primary education } \\
\text { Secondary education } \\
\text { University education }\end{array}$ & $\begin{array}{l}107 \\
55 \\
25 \\
18 \\
8\end{array}$ & $\begin{array}{l}50.2 \\
25.8 \\
11.7 \\
8.5 \\
3.8\end{array}$ & $\begin{array}{l}124 \\
46 \\
5 \\
5 \\
1 \\
\end{array}$ & $\begin{array}{l}68.5 \\
25.3 \\
2.8 \\
2.8 \\
0.6\end{array}$ & $\begin{array}{l}X^{2}=25.750 \\
\mathbf{M C}_{\mathrm{p}}<0.0001^{*}\end{array}$ \\
\hline $\begin{array}{l}\text { Marital status } \\
\text { Married } \\
\text { Divorced/single } \\
\text { Widow }\end{array}$ & $\begin{array}{l}162 \\
2 \\
49\end{array}$ & $\begin{array}{l}76.1 \\
0.9 \\
23.0\end{array}$ & $\begin{array}{l}69 \\
12 \\
100 \\
\end{array}$ & $\begin{array}{l}38.2 \\
6.6 \\
55.2 \\
\end{array}$ & $\begin{array}{l}X^{2}=59.840 \\
\mathbf{M C}_{\mathrm{p}<0.0001 *}<0\end{array}$ \\
\hline $\begin{array}{l}\text { Number of children } \\
\text { No children } \\
\text { From } 1-3 \\
\text { From } 4-6 \\
\text { More than } 6\end{array}$ & $\begin{array}{l}4 \\
119 \\
85 \\
5\end{array}$ & $\begin{array}{l}1.9 \\
55.9 \\
39.9 \\
2.3 \\
\end{array}$ & $\begin{array}{l}25 \\
57 \\
85 \\
14\end{array}$ & $\begin{array}{l}13.8 \\
31.5 \\
47.0 \\
7.7\end{array}$ & $\begin{array}{l}X^{2}=38.969 \\
p<0.0001 *\end{array}$ \\
\hline $\begin{array}{l}\text { Accompanying house hold } \\
\text { Alone } \\
\text { Not alone }\end{array}$ & $\begin{array}{l}8 \\
205 \\
\end{array}$ & $\begin{array}{l}3.8 \\
96.2\end{array}$ & $\begin{array}{l}63 \\
118 \\
\end{array}$ & $\begin{array}{l}34.8 \\
65.2\end{array}$ & $\begin{array}{l}X^{2}=63.860 \\
p<0.0001^{*}\end{array}$ \\
\hline $\begin{array}{l}\text { Have enough salary } \\
\text { Enough } \\
\text { Not enough }\end{array}$ & $\begin{array}{l}{[\mathrm{n}=177]} \\
107 \\
70\end{array}$ & $\begin{array}{l}60.5 \\
39.5\end{array}$ & $\begin{array}{l}{[n=145]} \\
80 \\
65\end{array}$ & $\begin{array}{l}55.2 \\
44.8 \\
\end{array}$ & $\begin{array}{l}X^{2}=0.912 \\
p=0.339\end{array}$ \\
\hline $\begin{array}{l}\text { chronic diseases } \\
\text { No } \\
\text { Yes } \\
\end{array}$ & $\begin{array}{l}48 \\
165 \\
\end{array}$ & $\begin{array}{l}22.5 \\
77.5 \\
\end{array}$ & $\begin{array}{l}19 \\
162 \\
\end{array}$ & $\begin{array}{l}10.5 \\
89.5 \\
\end{array}$ & $\begin{array}{l}X^{2}=10.047 \\
p=0.002 *\end{array}$ \\
\hline
\end{tabular}

$X^{2}:$ Chi-Square test ${ }^{M C} P:$ Monte Carlo corrected $P$-value ${ }^{*}$ significant at $P \leq 0.05$ 
Table (5): Relation between the studied elderly reported exposing to neglect and their socio-demographic characteristics $(n=394)$

\begin{tabular}{|c|c|c|c|c|c|}
\hline \multirow{3}{*}{$\begin{array}{l}\text { Socio-demographic } \\
\text { characteristics }\end{array}$} & \multicolumn{4}{|c|}{ Neglect } & \multirow{3}{*}{$\begin{array}{l}\text { Significance } \\
\text { tests }\end{array}$} \\
\hline & \multicolumn{2}{|c|}{ No $(n=340)$} & \multicolumn{2}{|c|}{ Yes $(n=54)$} & \\
\hline & No. & $\%$ & No. & $\%$ & \\
\hline $\begin{array}{l}\text { Age (years) } \\
60- \\
65- \\
70- \\
75- \\
\geq 80\end{array}$ & $\begin{array}{l}103 \\
122 \\
69 \\
28 \\
18\end{array}$ & $\begin{array}{l}30.3 \\
35.9 \\
20.3 \\
8.2 \\
5.3\end{array}$ & $\begin{array}{l}16 \\
14 \\
12 \\
4 \\
8\end{array}$ & $\begin{array}{l}29.6 \\
25.9 \\
22.2 \\
7.4 \\
14.9\end{array}$ & $\begin{array}{l}X^{2}=7.870 \\
p=0.096\end{array}$ \\
\hline $\begin{array}{l}\text { Gender } \\
\text { Male } \\
\text { Female } \\
\end{array}$ & $\begin{array}{l}184 \\
156 \\
\end{array}$ & $\begin{array}{l}54.1 \\
45.9 \\
\end{array}$ & $\begin{array}{l}25 \\
29 \\
\end{array}$ & $\begin{array}{l}46.3 \\
53.7\end{array}$ & $\begin{array}{l}X^{2}=1.144 \\
p=0.285\end{array}$ \\
\hline $\begin{array}{l}\text { Educational level } \\
\text { Illiterate } \\
\text { Read \& Write } \\
\text { Primary education } \\
\text { Secondary education } \\
\text { University education }\end{array}$ & $\begin{array}{l}195 \\
87 \\
27 \\
22 \\
9\end{array}$ & $\begin{array}{l}57.4 \\
25.6 \\
7.9 \\
6.5 \\
2.6 \\
\end{array}$ & $\begin{array}{l}36 \\
14 \\
3 \\
1 \\
0\end{array}$ & $\begin{array}{l}66.6 \\
25.9 \\
5.6 \\
1.9 \\
0.0\end{array}$ & $\begin{array}{l}X^{2}=4.172 \\
{ }^{M C} \\
p=0.374\end{array}$ \\
\hline $\begin{array}{l}\text { Marital status } \\
\text { Married } \\
\text { Divorced/single } \\
\text { Widow }\end{array}$ & $\begin{array}{l}210 \\
13 \\
117 \\
\end{array}$ & $\begin{array}{l}61.8 \\
3.8 \\
34.4 \\
\end{array}$ & $\begin{array}{l}21 \\
1 \\
32 \\
\end{array}$ & $\begin{array}{l}38.9 \\
1.9 \\
59.2 \\
\end{array}$ & $\begin{array}{l}\mathrm{X}^{2}=12.280 \\
\mathrm{MC}_{\mathrm{p}}=0.002 *\end{array}$ \\
\hline $\begin{array}{l}\text { Number of children } \\
\text { No children } \\
\text { From } 1-3 \\
\text { From } 4-6 \\
\text { More than } 6\end{array}$ & $\begin{array}{l}24 \\
159 \\
145 \\
12\end{array}$ & $\begin{array}{l}7.1 \\
46.8 \\
42.6 \\
3.5\end{array}$ & $\begin{array}{l}5 \\
17 \\
25 \\
7\end{array}$ & $\begin{array}{l}9.3 \\
31.5 \\
46.2 \\
13.0\end{array}$ & $\begin{array}{l}X^{2}=11.486 \\
p=0.011^{*}\end{array}$ \\
\hline $\begin{array}{l}\text { Accompanying house hold } \\
\text { Alone } \\
\text { Not alone }\end{array}$ & $\begin{array}{l}50 \\
290\end{array}$ & $\begin{array}{l}14.7 \\
85.3\end{array}$ & $\begin{array}{l}21 \\
33\end{array}$ & $\begin{array}{l}38.9 \\
61.1\end{array}$ & $\begin{array}{l}X^{2}=18.450 \\
p<0.0001 *\end{array}$ \\
\hline $\begin{array}{l}\text { Have enough salary } \\
\text { Enough } \\
\text { Not enough }\end{array}$ & $\begin{array}{l}{[\mathrm{n}=279]} \\
169 \\
110\end{array}$ & $\begin{array}{l}60.6 \\
39.4 \\
\end{array}$ & $\begin{array}{l}{[\mathrm{n}=43]} \\
18 \\
25\end{array}$ & $\begin{array}{l}41.9 \\
58.1 \\
\end{array}$ & $\begin{array}{l}X^{2}=5.358 \\
p=0.021 *\end{array}$ \\
\hline $\begin{array}{l}\text { chronic diseases } \\
\text { No } \\
\text { Yes } \\
\end{array}$ & $\begin{array}{l}63 \\
277 \\
\end{array}$ & $\begin{array}{l}18.5 \\
81.5\end{array}$ & $\begin{array}{l}4 \\
50 \\
\end{array}$ & $\begin{array}{l}7.4 \\
92.6 \\
\end{array}$ & $\begin{array}{l}X^{2}=4.084 \\
p=0.043^{*}\end{array}$ \\
\hline
\end{tabular}

$X^{2}:$ Chi-Square test ${ }^{M C} P:$ Monte Carlo corrected $P$-value ${ }^{*}$ significant at $P \leq 0.05$ 
Table (6): Relation between the studied elderly reported exposing to financial domestic exploit and their socio-demographic characteristics $(n=394)$

\begin{tabular}{|c|c|c|c|c|c|}
\hline \multirow{3}{*}{ Socio-demographic characteristics } & \multicolumn{4}{|c|}{$\begin{array}{l}\text { Exposure to financial domestic } \\
\text { exploit }\end{array}$} & \multirow{3}{*}{$\begin{array}{l}\text { Significance } \\
\text { Tests }\end{array}$} \\
\hline & \multicolumn{2}{|c|}{ No $(n=354)$} & \multicolumn{2}{|c|}{ Yes $(n=40)$} & \\
\hline & No. & $\%$ & No. & $\%$ & \\
\hline $\begin{array}{l}\text { Age (years) } \\
60- \\
65- \\
70- \\
75- \\
\geq 80\end{array}$ & $\begin{array}{l}102 \\
125 \\
73 \\
31 \\
23\end{array}$ & $\begin{array}{l}28.8 \\
35.3 \\
20.6 \\
8.8 \\
6.5\end{array}$ & $\begin{array}{l}17 \\
11 \\
8 \\
1 \\
3\end{array}$ & $\begin{array}{l}42.5 \\
27.5 \\
20.0 \\
2.5 \\
7.5\end{array}$ & $\begin{array}{l}X^{2}=4.658 \\
{ }^{M C} \\
p=0.319\end{array}$ \\
\hline $\begin{array}{l}\text { Gender } \\
\text { Male } \\
\text { Female } \\
\end{array}$ & $\begin{array}{l}190 \\
164\end{array}$ & $\begin{array}{l}53.7 \\
46.3 \\
\end{array}$ & $\begin{array}{l}19 \\
21\end{array}$ & $\begin{array}{l}47.5 \\
52.5\end{array}$ & $\begin{array}{l}X^{2}=0.550 \\
p=0.458\end{array}$ \\
\hline $\begin{array}{l}\text { Educational level } \\
\text { Illiterate } \\
\text { Read \& Write } \\
\text { Primary education } \\
\text { Secondary education } \\
\text { University education }\end{array}$ & $\begin{array}{l}207 \\
93 \\
28 \\
20 \\
6\end{array}$ & $\begin{array}{l}58.5 \\
26.3 \\
7.9 \\
5.6 \\
1.7\end{array}$ & $\begin{array}{l}24 \\
8 \\
2 \\
3 \\
3\end{array}$ & $\begin{array}{l}60.0 \\
20.0 \\
5.0 \\
7.5 \\
7.5\end{array}$ & $\begin{array}{l}X^{2}=6.478 \\
{ }^{M C} \\
p=0.158\end{array}$ \\
\hline $\begin{array}{l}\text { Marital status } \\
\text { Married } \\
\text { Divorced/single } \\
\text { Widow }\end{array}$ & $\begin{array}{l}212 \\
10 \\
132 \\
\end{array}$ & $\begin{array}{l}59.9 \\
2.8 \\
37.3 \\
\end{array}$ & $\begin{array}{l}19 \\
4 \\
17\end{array}$ & $\begin{array}{l}47.5 \\
10.0 \\
42.5 \\
\end{array}$ & $\begin{array}{l}X^{2}=6.410 \\
p=0.041^{*}\end{array}$ \\
\hline $\begin{array}{l}\text { Number of children } \\
\text { No children } \\
\text { From } 1-3 \\
\text { From } 4-6 \\
\text { More than } 6 \\
\end{array}$ & $\begin{array}{l}23 \\
161 \\
153 \\
17 \\
\end{array}$ & $\begin{array}{l}6.5 \\
45.5 \\
43.2 \\
4.8 \\
\end{array}$ & $\begin{array}{l}6 \\
15 \\
17 \\
2 \\
\end{array}$ & $\begin{array}{l}15.0 \\
37.5 \\
42.5 \\
5.0\end{array}$ & $\begin{array}{l}X^{2}=4.050 \\
{ }^{2} C_{p}=0.260\end{array}$ \\
\hline $\begin{array}{l}\text { Accompanying house hold } \\
\text { Alone } \\
\text { Not alone }\end{array}$ & $\begin{array}{l}59 \\
295\end{array}$ & $\begin{array}{l}16.7 \\
83.3\end{array}$ & $\begin{array}{l}12 \\
28\end{array}$ & $\begin{array}{l}30.0 \\
70.0\end{array}$ & $\begin{array}{l}X^{2}=4.32 \\
p=0.038 *\end{array}$ \\
\hline $\begin{array}{l}\text { Have enough salary }[\mathbf{n}=\mathbf{3 2 2}] \\
\text { Enough } \\
\text { Not enough }\end{array}$ & $\begin{array}{l}{[\mathrm{n}=186]} \\
166 \\
20\end{array}$ & $\begin{array}{l}89.2 \\
10.8\end{array}$ & $\begin{array}{l}{[\mathrm{n}=36]} \\
21 \\
15\end{array}$ & $\begin{array}{l}58.3 \\
41.7\end{array}$ & $\begin{array}{l}X^{2}=0.001 \\
p=0.973\end{array}$ \\
\hline $\begin{array}{l}\text { chronic diseases } \\
\text { No } \\
\text { Yes }\end{array}$ & $\begin{array}{l}63 \\
291 \\
\end{array}$ & $\begin{array}{l}17.8 \\
82.2\end{array}$ & $\begin{array}{l}4 \\
36 \\
\end{array}$ & $\begin{array}{l}10.0 \\
90.0\end{array}$ & $\begin{array}{l}X^{2}=1.548 \\
p=0.213\end{array}$ \\
\hline
\end{tabular}


Table (7): Relation between the studied elderly reported exposing to sexual domestic violence and their socio-demographic characteristics $(n=394)$

\begin{tabular}{|c|c|c|c|c|c|}
\hline \multirow{3}{*}{ Socio-demographic characteristics } & \multicolumn{4}{|c|}{ Exposing to sexual domestic violence } & \multirow{3}{*}{ Significance } \\
\hline & \multicolumn{2}{|c|}{ No $(n=383)$} & \multicolumn{2}{|c|}{ Yes $(n=11)$} & \\
\hline & No. & $\%$ & No. & $\%$ & \\
\hline \multicolumn{6}{|l|}{ Age (years) } \\
\hline $60-$ & 113 & 29.5 & 6 & 54.5 & \multirow{5}{*}{$\begin{array}{l}X^{2}=5.583 \\
{ }^{M C}=0.210\end{array}$} \\
\hline $65-$ & 132 & 34.5 & 4 & 36.4 & \\
\hline $70-$ & 81 & 21.1 & 0 & 0.0 & \\
\hline $75-$ & 32 & 8.4 & 0 & 0.0 & \\
\hline$\geq 80$ & 25 & 6.5 & 1 & 9.1 & \\
\hline \multirow{3}{*}{$\begin{array}{l}\text { Gender } \\
\text { Male } \\
\text { Female }\end{array}$} & & & & & \multirow{3}{*}{$\begin{array}{l}X^{2}=5.522 \\
p=0.019 *\end{array}$} \\
\hline & 207 & 54.0 & 2 & 18.2 & \\
\hline & 176 & 46.0 & 9 & 81.8 & \\
\hline \multicolumn{5}{|l|}{ Educational level } & \multirow{6}{*}{$\begin{array}{l}\mathrm{X}^{2}=1.580 \\
{ }^{\mathrm{MC}} \mathrm{p}=0.815\end{array}$} \\
\hline Illiterate & 223 & 58.2 & 8 & 72.7 & \\
\hline Read \& Write & 99 & 25.8 & 2 & 18.2 & \\
\hline Primary education & 29 & 7.6 & 1 & 9.1 & \\
\hline Secondary education & 23 & 6.1 & 0 & 0.0 & \\
\hline University education & 9 & 2.3 & 0 & 0.0 & \\
\hline \multicolumn{5}{|l|}{ Marital status } & \multirow{4}{*}{$\begin{array}{l}\mathrm{X}^{2}=21.91 \\
{ }^{\mathrm{MC}} \mathrm{p}<0.0001 *\end{array}$} \\
\hline Married & 229 & 59.8 & 2 & 18.2 & \\
\hline Divorced/single & 11 & 2.9 & 3 & 27.3 & \\
\hline Widow & 143 & 37.3 & 6 & 54.5 & \\
\hline \multicolumn{5}{|l|}{ Number of children } & \multirow{5}{*}{$\begin{array}{l}\mathrm{X}^{2}=2.523 \\
\mathrm{MC}_{\mathrm{p}}=0.428\end{array}$} \\
\hline No children & 27 & 7.1 & 2 & 18.1 & \\
\hline From 1-3 & 172 & 44.9 & 4 & 36.4 & \\
\hline From 4-6 & 166 & 43.3 & 4 & 36.4 & \\
\hline More than 6 & 18 & 4.7 & 1 & 9.1 & \\
\hline \multicolumn{5}{|l|}{ Accompanying house hold } & \multirow{3}{*}{${ }^{\mathrm{FE}} \mathrm{p}=0.425$} \\
\hline Alone & 68 & 17.8 & 3 & 27.3 & \\
\hline Not alone & 315 & 82.2 & 8 & 72.7 & \\
\hline Have enough salary [n=322] & {$[\mathrm{n}=312]$} & & {$[\mathrm{n}=10]$} & & \multirow{3}{*}{${ }^{\mathrm{FE}} \mathrm{p}=0.101$} \\
\hline Enough & 184 & 58.9 & 3 & 30.0 & \\
\hline Not enough & 128 & 41.1 & 7 & 70.0 & \\
\hline \multicolumn{5}{|l|}{ chronic diseases } & \multirow{3}{*}{${ }^{\mathrm{FE}} \mathrm{p}=0.698$} \\
\hline No & 66 & 17.2 & 1 & 9.1 & \\
\hline Yes & 317 & 82.8 & 10 & 90.9 & \\
\hline
\end{tabular}




\section{DISCUSSION:}

The phenomenon of domestic violence due to of its multi-faceted consequences is regarded not only as a significant individual problem but also as an important social problem. The costs faced by society as a result of domestic violence are enormous including individual, social problems and even financial expenses (American Psychological Association, 2014). Domestic violence affects every age group and is present throughout the life span, it is a major cause of death and disability especially among elderly. Domestic violence is a violence that occurs in home. It is defined as the willful infliction of pain, injury, debilitating mental anguish, unreasonable confinement and deprivation by a caregiver. Domestic violence occurs when one person in an intimate relationship behaves in a way that causes fear and harm to another person (O'Brien, et al., 2013).

Regarding the elderly's knowledge about types of domestic violence, the present study findings showed that about two-fifth of the elderly considered that the most common type of domestic violence was physical. This result was similar to a study in Korea conducted by Lee, et al., (2014) who found that there was a low perception about the type of domestic violence among elderly. This might be due to decrease the awareness about elderly domestic violence.

The present study reported that there was a statistically significant associations between having chronic disease and physical domestic violence. These results were congruent with the study done by Policastro \& Finn, (2017) in United States of America who found that older adults who had poor health were more likely to experience physical domestic violence at age 60 years or older. This might be due to chronic diseases increased independency of older people.

The present study reported that there was no statistical significant associations between physical domestic violence and gender. This result was congruent with the result of a study done by Johnson, et al., (2014) who reported that the victim's sex had no significant influence on the likelihood of experiencing physical domestic violence. But Guedes, et al., (2015) reported that elderly women were more liable to be abused than men.

The present study reported that there was a statistical significant associations between age and marital status and emotional domestic violence. This result was similar 
to the result of Guedes, et al., (2015) who reported that age and marital status experienced more psychological violence perpetrated by family members. This might be due to increase age is associated with increased elderly violence and elderly people became more sensitive to any thing.

The present study reported that there was a statistical significant associations between level of education and emotional domestic violence. This was in harmony with Naughton, et al., (2012) who stated that there was an inverse relationship between elderly emotional domestic violence and level of education this might be due to increase percentage of parent illiterately while the rate in present study was about three-fifth.

The present study reported that there was a statistical significant associations between accompanying household and emotional domestic violence. This result was similar to the result of a study carried out in India by Wu, et al., (2012) who observed that elderly people who were living alone had associated with an increased risk of emotional violence. However Dong \& Simon (2013) reported that there wasn't statistical significant association with the number of persons living in the household and elderly emotional violence.

The present study reported that there was a statistical significant associations between chronic diseases and emotional domestic violence. These result were similar to the results of a study conducted by $\mathrm{Wu}$, et al., (2012), who stated that physical disability and chronic illness were associated with increased risk of emotional domestic violence. While Dong \& Simon (2010) identified a negative statistical association between chronic diseases and emotional domestic violence also they found that impairment in physical function and indicators of activities of daily living and instrumental activities of daily living were not independently associated with the increased risk of elder emotional domestic violence.

The present research reported that there was a statistical significant relation between neglect and marital status. This result was similar to a result of a study conducted in Ireland by Naughton, et al., (2010) who reported that the highest level of elderly violence and neglect occurred in people who were divorced or separated. People who were widowed had the second highest risk of elderly domestic violence, while older people who were single (never married) reported the lowest level of mistreatment. This 
might be due to they considered that presence of man protect them from danger or this might be due to loneliness.

The present study reported that there was a statistical significant relation between neglect and accompanying house hold. This result was in harmony with a study conducted by Gerstel, (2011) who reported that there was statistical significant relation between neglect and accompanying household . Also Naughton, et al., (2010) reported that having a close supportive family was universally endorsed as a protective factor against all kinds of elderly violence. The highest levels of elderly violence occurred in a complex households in particular where one older person lived in an intergenerational household.

The present study reported that there was a statistical significant relation between neglect and having enough salary. This result was similar to the result of a study conducted by Abbas, et al., (2010) who reported that the economic situation of elderly people seemed to be a factor in elder violence and neglect. This might be due to decrease economic condition in these villages.

The present study reported that there wasn't statistical significant relation between neglect and level of education. This result wasn't in harmony with the study conducted in Canada by Lai, (2011) who found that lower education levels had consistently emerged as a risk factor for elderly violence and neglect. Also, Sebastian \& Sekher, (2011) in India found that illiterate people were three times more likely to be abused while 5 years or less of schooling was also associated with an increased risk of elderly violence.

This study found that there was a statistical significant relation between financial domestic violence and accompanying household. This result was similar to the study in New York city which conducted by Janey, et al., (2014) who reported that there was combination with the higher prevalence of social isolation and decrease household members and financial domestic violence. While these results weren't in agreement with those of the study conducted by Bavel, et al., (2010) who stated that some extended family units involving three generations living in the same household had high levels of interdependence with frequent exchanges of financial and social support that might reduce exposure of financial domestic violence. 
The present study reported that there was a statistical significant associations between marital status and financial domestic violence. These results were in agreement with those of the study conducted by Bavel, et al., (2010) who reported in their study about elder violence that there was an association between marital status and financial domestic violence.

This study found that there was a statistical significant relation between gender and sexual domestic violence, This was similar to the study in New York city which conducted by Bows, \& Westmarland, (2017) in the United Stat of America who found that sexual violence against older people represented the vast majority of victims were females. Also Cannell, et al., (2014) in a study in the United State of America found that there was a significant relation between gender and sexual violence with a stronger association among women. Also these results were incongruent with a study done by Allender, et al., (2014) who found a statistically significant relation between gender and sexual domestic violence. Older women experienced sexual violence at a higher rate than elderly men $67.0 \%$ of elderly women while only $32.0 \%$ of elderly men experienced sexual violence. In contrast, other studies haven't found any difference between gender and sexual elder violence (Laumann, et al., 2008).

This study found that there was a statistical significant relation between marital status and sexual violence. This result was similar to the result of a study carried out among the older Chinese people who had been sexually abused were more likely to be currently unmarried (Wu, et al., 2012). In both India and Korea, being a widow was associated with an increased risk of sexual violence (Kim, et al., 2012). In other studies conducted by (Dong \& Simon, 2013) found that marital status wasn't associated with any kind of elderly violence.

\section{CONCLUSION:}

\section{Based on the present study findings, it can be concluded that :}

The most elderly people have lack of knowledge about the definition, types and causes of violence. The most common forms of domestic violence suffered by elderly people was emotional domestic violence, while the least of which was sexual violence. There were statistical significant associations between exposure to physical domestic violence, neglect, with having chronic diseases and take medication on time. while there were statistical significant associations between elderly who are exposed to emotional 
domestic violence and all socio-demographic characteristic except the item of have enough salary. Also there were statistical significant relation between exposure to financial exploit and accompanying house hold .In addition there were statistical significant relation between elderly exposed to sexual violence, gender, marital status and take medication on time.

\section{RECOMMENDATIONS:}

Based on the results of the present study, the following recommendations were suggested:

- Increasing elderly awareness and knowledge about elderly domestic violence through mass media and elderly class .

- Educating the caregivers is the cornerstone for preventing elder domestic violence by focusing on the special needs and problems of the elderly and about the risk factors for violence.

- Community campaigns using various educational media are essential to increase knowledge and awareness about domestic violence among all population in order to identify risk factors of domestic violence .

- Conducting further studies for development of practice guidelines for community health nurse and standards of care for elderly in order to achieve, maintain, and advance good quality health services. These guidelines should be clearly written .

\section{REFERENCES:}

Abbas , H. , Sayed, M., Taha ,M.,\& Mohamad, E. (2010) : study on Frequency and Risk Factors of Elder Abuse in the University Hospital and Health Insurance Attendees in Ismailia City . Cairo University . Faculty of Medicine. master degree . Medical Journal . 78 (1): pp. 29-33 available at www.medicaljournalofcairouniversity.com

Abdel-Rahman T. \& El-Gaafary M. (2012): Study on elder mistreatment in a rural area in Egypt. Geriatrics and gerontology international journal. 12(3): pp. 532-537. Available at http://onlinelibrary.wiley.com/doi/10.1111/j.14470594.2011.00780.x/abstract.

Administration on Aging (2012): Aging statistics. available at http://www.aoa.gov/AoARoot/Aging_Statistics/index.aspx. 
Allender ,J. ,Rector ,C.\& Warner , K. (2014) : Community Health Nursing Promoting and Protecting the Public's Health .Wolters Kluwer Lippincott Williams \&Wilkins China . $8^{\text {th }}$ edition. pp. 57- 60 .

American Psychological Association (2014) : Older Adults' Health and AgeRelated Changes available at http://www.apa.org/pi/aging/resources/guides/older.aspx.

Barnes, J., TenEyck, M., Boutwell, B. \& Beaver, K. (2013): Indicators of domestic/intimate partner violence are structured by genetic and non shared environmental influences. Journal of psychiatric research. 47(3) : pp. 371-376.

Bavel, M., Janssens, K., Schakenraad, W. \& Thurlings, N. (2010) : Elder abuse in Europe: Background and position paper. Utrecht. The Netherlands: MOVISIE, Netherlands Centre for Social Development. The European reference Framework . online for the prevention of Elder abuse and Neglect .14 (22) : pp. 1-72 .

Bows, H., \& Westmarland, N. (2017): Rape of older people in the United Kingdom: challenging the Real-rape stereotype. The British Journal of Criminology. 57(1) : pp. 1-17.

Cannell, M., Manini, T., Almaguer, E., Molina, M.,\& Andresen, E. (2014) : US population estimates and correlates of sexual abuse of community-dwelling older adults. Journal of Elder Abuse and Neglect . 26 (4) : pp. 398 - 413.

Dong, X., \& Simon, M. (2010) : Is impairment in physical function associated with increased risk of elder mistreatment? Findings from a community-dwelling Chinese population. Journal of Public Health. 125(12) : pp. 743-753.

Dong, X., \& Simon, M. (2013) : Urban and rural variations in the characteristics associated with elder mistreatment in a community-dwelling Chinese population. Journal of Elder Abuse \& Neglect. 25 (2): pp. 97-125.

Fulmer, T. (2003). Elder abuse and neglect assessment. Journal of Gerontological Nursing . 29(6) : p. 4-5.

Gerstel, N. (2011). Rethinking families and community: The color, class, and centrality of extended kin ties. Journal of Sociological forum . 26(1) : pp. 1-20.

Guedes, D., Alvarado, B., Phillips, S., Curcio, C ., Zunzunegui, M. \& Guerra, R. (2015) : Socioeconomic status, social relations and domestic violence (DV) against 
elderly people in Canada, Albania, Colombia and Brazil. Archives of gerontology and geriatrics. 60(3) : pp. 492-500.

Janey C. ,David P.,Paul L. Charles R. ,Martin T.,Karl A. \&Mark S. (2014) : Financial Exploitation of Older Adults: A Population Based Prevalence Study. journal of General Internal Medicine. 29 (12) : pp. 1615-1623

Johnson M. , Leone J. \& Xu Y. (2014): Intimate terrorism and situational couple violence in general surveys: Ex-spouses required. Journal of Violence Against Women . 20 ( 3) : pp. 186-207.

Killick, C., Taylor, B., Begley, E., Carter, J., \& O’Brien, M. (2015): Older people's conceptualization of abuse: A systematic review. Journal of elder abuse \& neglect. 27(2): pp. 100-120.

Kim, O., Jeon , H. \& Kim, B. (2012) : The relating factors of elder abuse among community dwelling elderly with stroke . Korean Journal of Adult Nursing . 24 ( 3) : pp. 466-476.

Lai, D., (2011). Abuse and neglect experienced by aging Chinese in Canada. Journal of Elder Abuse \& Neglect. 23(4): pp. 326-347.

Laumann, E., Leitsch, S. \& Waite, L. (2008) : Elder mistreatment in the United States: prevalence estimates from a nationally representative study . Journal of Gerontology . 63 ( 4): pp. 48-54.

Lee, H., Yoon , H., Yoon, J., Kwon, J., Park, E., Nam, R. \& Park, K. (2014) : Perception and help-seeking intention of intimate partner violence in later life: An international perspective. Journal of Aggression, Maltreatment \& Trauma . 23(1) : pp. 45-66.

Naughton, C., Drennan, J., Treacy, M., Lafferty, A., Lyons, I., Phelan, A., Quin, S.,O’Loughlin, A.\& Delaney, L. (2010) : study on Abuse and Neglect of Older People in Ireland: Report on the National Study of Elder Abuse and Neglect. National Centre for the Protection of Older People. University College Dublin. Belfield, Dublin 4, Ireland .pp. $1-120$. 
Naughton, C., Drennan, J., Lyons, I., Lafferty, A., Treacy, M.,Phelan, A. \& Delaney, L. (2012) : Elder abuse and neglect in Ireland: results from a national prevalence survey. Journal of Age \& Ageing . 41(1) : pp. 98-103.

Natalie S. (2013): Queering international human rights: LGBT access to domestic violence remedies . Journal of Gender, Social Policy \& the Law. American University. 21 (3) : pp. 583-607.

O’Brien M., Begley, E., Carter Anand, J., Killick, C., Taylor, B., Doyle, E., McCarthy, M., McCrossan, S. \& Moran, E. (2011) :Study on A total indifferent to our dignity . Older people's understandings of elder abuse. Age Action Ireland and CARDI, Dublin. Faculty of Social Sciences. School of Sociology and Applied Social Studies Faculty of Social Sciences. P. 92.

Penhale, B.( 2010): Responding and intervening in elder abuse and neglect. Ageing International Journal. 35 (3) : pp. 235-252.

Policastro, C., \& Finn, M. (2017) : Coercive control and physical violence in older adults: Analysis using data from the National Elder Mistreatment Study. Journal of interpersonal violence . 32(3) : pp. 311-330.

Sebastian, D., \& Sekher, T. (2011) : Extent and nature of elder abuse in Indian families: A study in Kerala. Help Age India-Research and Development Journal . 17 (3): pp. 20-28.

WHO (2011): Definition of an older or elderly person Proposed Working Definition of an Older Person in Africa for the MDS .Project available at http://www.who.int/healthinfo/survey/ageingdefnolder/en/index.h tml.

Wu, L., Chen, H., Xiang, H. , Zhang, T. \& Wang, Y.(2012) : Prevalence and associated factors of elder mistreatment in a rural community in People's Republic of China: a cross-sectional study. PLOS One Tenth Anniversary.7(2) : p. 33857. United States. available at

http://www.plosone.org/article/info:doi/10.1371/journal.pone.0033857. 
تقييم العنف الاسري ضد المسنين فـي مـديــــة المـنصــورة

نورا السيد محمود محمد ـ أ. د/ نجاة سعيد حبيب ـ ا. م. د/ نجلاء ابراهيم محمد غيدة ـ ا. م. د/ مها موسى محمد موسى

بكالوريوس تمريض__جامعة المنصورة، أستاذ تمريض صحة المجتمع _ـلية التمريض_جامعة القاهرة، استاذ مساعد تمريض صحة الأسرة و المجتمع _ كلية التمريض__ جامعة بورسعيد ، استاذ مساعد تمريض صحة الأسرة و المجتمع _ _كلية التمريض__ جامعة بورسعيد .

\section{الــــاصــة}

العنف الأسري ضد المسنين يحدث عندما يتعرض المسن للخوف أو للضرر من قبل اي شخص يوجد بينه وبين المسن صلة قرابة و يشمل أنواع كثيرة منها العنف النفسي والجسدي والجنسي و والاهمال وأيضـا العنف المالي. الـهــف مــن الــر ســـة: تهدف هذه الدراسة الي تقييم العنف المنزلي ضد المسنين في مدينة المنصورة .نـوع الــراسة: دراسة وصفية.مكان الدراسة: في العيادات الباطنة الخارجية من ثلاث مر اكز صحيه هى منية النصر وشربين و أجا والتي تم اختيار ها عشو ائيا من ضمن 10 مركز من مدينة المنصورة. العينة: عينة قصدية من المسنين

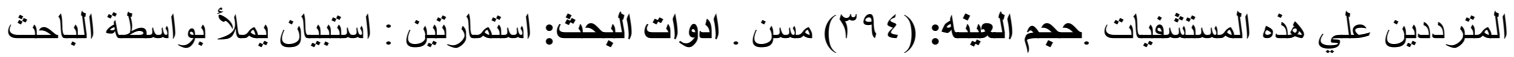
لتقييم معلومات المسن عن العنف ومعرفة مدي تعرضهم للعنف الاسري .استبيان لتقييم اي من أعراض العنف

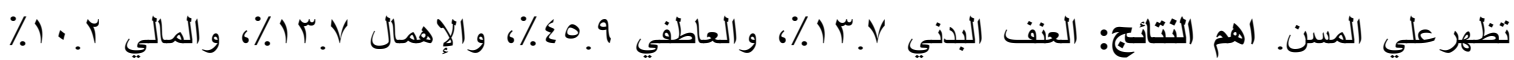
و الجنس ^. ؟٪ ـ الملخص:لقد لوحظ من هذه الدر اسة قلة معلومات المسنين عن العنف المنزلي , كما وجد ان العنف النفسي هو اكثر الانواع شيو عا بينهم ـ. لذا نوصي بذلك: بزيادة وعي المجتمع حول إساءة معاملة المسنين عن طريق استخدام وسائل الاعلام.

الكلمات المرشدة: العنف الجسدي ـ العنف النفسي _ العنف المالي _ العنف الجنسي_ الاهمال . 